\title{
Ground Anthrax Bacillus Refined Isolation (GABRI) method for analyzing environmental samples with low levels of Bacillus anthracis contamination
}

\author{
Antonio Fasanella1*, Pietro Di Taranto1, Giuliano Garofolo², Valeriana Colao³, Leonardo Marino1, \\ Domenico Buonavoglia ${ }^{3}$, Carmine Pedarra', Rosanna Adone ${ }^{4}$ and Martin Hugh-Jones ${ }^{5}$
}

\begin{abstract}
Background: In this work are reported the results of a qualitative analytical method capable of detecting Bacillus anthracis spores when they are present in very low concentration in the soil. The Ground Anthrax Bacillus Refined Isolation (GABRI) method, assessed in our laboratory, was compared with the classic method. The comparison involved artificially anthrax-contaminated soil samples (500 spores/7.5 grams soil) and naturally contaminated soil samples collected in Bangladesh during a field investigation.

Results: The results indicated that, in contrast to the classic method, the GABRI method was able to detect $B$. anthracis in all contaminated samples. The GABRI method produces a more sensitive measure of anthrax spore presence significantly different from the standard method. In particular, the latter is more sensitive to the presence of normal soil contaminants.

Conclusion: The main feature of the GABRI method is its ability to strongly reduce the presence of the environmental contaminants, which being much more numerous than $B$. anthracis tend to inhibit their germination and growth making it extremely difficult to visualize any colonies. The reduction of the microbial environment also allows one to be able to culture and test a larger quantity of potentially contaminated soil and to isolate $B$. anthracis when the spores are present in very low concentrations in the soil.
\end{abstract}

Keywords: Bacillus anthracis, Contaminated soil, Isolation, Method

\section{Background}

B. anthracis is the causative agent of anthrax, a noncontagious infectious disease that primarily affects herbivores. However, all mammals, including humans, can be involved. Though having almost completely disappeared in the industrialized countries, anthrax is an important public health problem in many Asian and African areas [1].

B. anthracis is a Gram positive, capsulated, and sporeforming bacterium. The spores are very robust and can survive in a suitable soil for several decades. In the Kruger National Park (Africa) B. anthracis spores have been isolated from animal bones estimated to be about 200 years old [2]. The ability of B. anthracis spores to survive outside the body is key for the ecology and evolution of this

\footnotetext{
* Correspondence: a.fasanella@izsfg.it

'Istituto Zooprofilattico Sperimentale della Puglia e della Basilicata, Anthrax Reference Institute of Italy, Foggia, Italy

Full list of author information is available at the end of the article
}

pathogen. Higgins [3], Minett \& Dhanda [4], Van Ness \& Stein [5] and Van Ness [6] observed that spores survive in soils rich in organic material and calcium and much better in alkaline soil with $\mathrm{pH}$ above 6.0 and a temperature of about $15^{\circ} \mathrm{C}$.

M. Hugh-Jones (unpublished data) noted that in Texas after heavy rains depressed areas, locally called 'pot holes', accumulate humus and minerals from the surrounding soil. The pot holes were found to have calcium concentrations 2-3 times higher, phosphorus 6-10 times and magnesium 2 times higher than the surrounding ground, and this creates locally favorable conditions to enable a better survival of spores in places with otherwise unfavourable soil, e.g., sandy loams [7]. However the strong hydrophobicity of the surface and the buoyancy of the spores have an important role in the ecology of the bacterium. Van Ness noted that the outbreaks of anthrax 
develop mainly during the dry months that follow a prolonged period of rain. These climatic aspects and the fact that the spores are characterized by a high floating capacity suggest that water plays an important role in the ecology of the bacterium. Rainwater, having washed away the surrounding ground, tends to collect in the low lying parts favoring the concentration of spores. This increases the probability that a grazing animal will acquire an infective dose of spores. However it takes time and special natural events to create sites of concentrations of spores which can cause new infections in grazing animals [6].

It is very easy to isolate $B$. anthracis from biological samples. It grows very well on sheep blood agar. The colonies are white, slightly opaque, a pasty consistency, non-haemolytic and margins slightly indented give the typical appearance to "caput medusae".

However the isolation from the soil is much more difficult than textbooks recount due to the presence of telluric contaminants such as yeasts and bacteria, especially spore-formers, closely related to $B$. anthracis, such as B. thuringiensis, B. cereus, B. mycoides [8]. The conflicting presence of contaminating bacteria makes it necessary to heat treat a sample to reduce the vegetative forms of this microbial load [9]. However, heat treatment is ineffective against spores closely related to $B$. anthracis, and this necessitates the use of selective medium [10]. Dragon and Rennie (2001) have shown that a selective culture medium is crucial when isolating $B$. anthracis from environmental samples. The "PLET medium" (Polymyxin, lysozyme, EDTA, thallium acetate) and subsequently the Anthracis Chromogenic Agar (CHRA) [10] can be considered semi-selective media that are able to inhibit the growth of several bacteria and encourage those bacilli belonging to the Cereus group.

While the cultivation and the direct isolation of the bacterium from environmental samples can be difficult and time-consuming compared to molecular methods, e.g. PCR, it is still considered the most sensitive method for the detection of $B$. anthracis in environmental samples [11]. In fact, the biomolecular methods based on the amplification of DNA extracted directly from the environmental sample are not very sensitive. It is known that the spores release their DNA with much difficulty and, furthermore, the examined sample may contain chemicals or organic substances that might interfere with the processes of amplification [12]. Finally, the sensitivity of this method is limited by the very small amount of extract which can be examined [11].

In this work we report the results of a qualitative analytical method capable of detecting very low levels of B. anthracis environmental contamination. We compare the Ground Anthrax Bacillus Refined Isolation (GABRI) method with the classic method as described in the OIE Terrestrial Manual 2012. The comparison involved artificially anthrax-contaminated soil samples as well as naturally contaminated soil samples collected in farms of Bangladesh that had suffered from confirmed outbreaks of anthrax [13].

\section{Methods}

\section{Ethics statement}

Experiments described in this paper, previously authorized by the Italian Ministry of Health, (DSVET 0003319-P-13/06/2011), have been conducted without using animals.

\section{Preparation of anthrax spores}

The pathogen strain A0843 of B. anthracis [14] was seeded on sporulation agar [15] and incubated at $37^{\circ} \mathrm{C}$ for 24 hours and then at $23^{\circ} \mathrm{C}$. Every 10 days it was tested to verify the level of sporulation and when it reached around 90\%, the spores were collected in a sterile saline solution. After three washes, the suspension was incubated at $56^{\circ} \mathrm{C}$ for $20 \mathrm{~min}$ to eliminate any residual vegetative forms.

\section{Preparation of artificially contaminated soil samples}

About 500 grams of soil were collected from the public gardens of the city of Foggia (Italy). The sample was tested and found negative for $B$. anthracis. Twelve aliquots of 7.5 grams each were prepared and 500 spores of the B. anthracis strain A0843 were added to each aliquot. Six aliquots were examined by the classic method and six aliquots were examined by the GABRI method.

\section{Naturally contaminated soil samples}

In December 2010, eight farms were visited in Bangladesh where there had been confirmed anthrax outbreaks earlier in the year [13]. Soil samples were collected from selected sites on these farms and were sent for analysis to the Reference Anthrax Institute (Foggia, Italy). The list of samples is reported in Table 1.

\section{Classic method for isolation of $B$. anthracis}

The method used for the isolation of spores from environmental samples was that described in OIE Terrestrial Manual 2012 [15], with some modifications. For culturing and isolation of $B$. anthracis the TSMP medium was used, consisting in the semi-selective Columbia blood agar added with trimethoprim $(16 \mathrm{mg} / \mathrm{lt})$, sulfamethoxazole $(80 \mathrm{mg} / \mathrm{lt})$, methanol $(5 \mathrm{ml} / \mathrm{lt})$ and polymyxin (300,000 units/lt). Based on our experience, TSMP has the same efficacy of PLET in isolating $B$. anthracis (data not shown). Briefly, to each 7.5 gram aliquot of soil sample were added $22.5 \mathrm{ml}$ of deionized sterile water. After 30 minutes of washing by vortexing, the suspension was incubated at $64^{\circ} \mathrm{C}$ for $20 \mathrm{~min}$ to eliminate any vegetative forms of soil contaminants [16]. 


\begin{tabular}{|c|c|c|c|c|c|c|c|c|}
\hline \multirow{3}{*}{$\begin{array}{c}\text { Soil sample } \\
\text { (Subdistricts of Bangladesh) }\end{array}$} & \multirow{2}{*}{\multicolumn{3}{|c|}{$\begin{array}{c}\begin{array}{c}\text { CFU of } B \text {. anthracis isolated } \\
\text { by classic method }\end{array} \\
\text { Total of } 10 \text { plates }\end{array}$}} & \multirow{2}{*}{\multicolumn{3}{|c|}{$\begin{array}{c}\text { CFU of contaminants } \\
\text { isolated by classic method } \\
\text { Total of } 10 \text { plates }\end{array}$}} & \multirow{2}{*}{\multicolumn{2}{|c|}{$\begin{array}{c}\text { CFU of } B \text {. anthracis and contaminants } \\
\text { isolated by GABRI method }\end{array}$}} \\
\hline & & & & & & & & \\
\hline & Undiluted & $1: 10$ & $1: 100$ & Undiluted & $1: 10$ & $1: 100$ & CFU of B. anthracis & CFU of contaminants \\
\hline Faridpur & 0 & 4 & 8 & 8482 & 2190 & 314 & 394 & 1622 \\
\hline Sapatul & 108 & 32 & 0 & 1380 & 162 & 22 & 256 & 200 \\
\hline Dhunot & 0 & 0 & 0 & 4404 & 598 & 60 & 10 & 1164 \\
\hline Santhia & 120 & 128 & 15 & 4968 & 826 & 90 & 10,000 & 276 \\
\hline Shahazadpur & 0 & 0 & 0 & 1074 & 100 & 14 & 10 & 280 \\
\hline Ullapara & 20 & 0 & 0 & 66 & 2 & 0 & 68 & 130 \\
\hline Shahazadpur & 2 & 0 & 0 & 426 & 44 & 2 & 12 & 176 \\
\hline Average & 35.7 & 23.4 & 3.3 & 2971.4 & 560.3 & 71.7 & 1535.7 & 549.7 \\
\hline
\end{tabular}

From each sample, $10 \mathrm{ml}$ of supernatant were collected and dilutions of 1:10 and 1:100 were made using normal saline solution.

Subsequently, 10 plates of TMSP were seeded with the undiluted suspension (100 $\mu \mathrm{l} /$ plate), 10 plates with the 1:10 dilution and 10 plates with the 1:100 dilution. After 24 and 48 hours of incubation at $37^{\circ} \mathrm{C}$, each plate was examined for the presence of suspect colonies of $B$. anthracis and of contaminants. All colonies were counted. B. anthracis colonies were identified by Gram staining, colony morphology and anthrax-specific PCRs [17].

\section{Ground anthrax bacillus refined isolation (GABRI) procedure}

To each 7.5 gram aliquot were added $22.5 \mathrm{ml}$ of washing buffer consisting of deionized water containing $0.5 \%$ Tween 20. After 30 minutes of washing by vortexing, the suspension was centrifuged at $2000 \mathrm{rpm}$ for $5 \mathrm{~min}$ to eliminate gross debris. The supernatant was harvested and then incubated, aerobically, at $64^{\circ} \mathrm{C}$ for $20 \mathrm{~min}$ to eliminate vegetative forms of B. anthracis. After incubation, $5 \mathrm{ml}$ of supernatant were added to $5 \mathrm{ml}$ of Tryptose Phosphate Broth containing $125 \mu \mathrm{g} / \mathrm{ml}$ of Fosfomycin. Then, from each sample, 10 plates of TMSP were seeded with $1 \mathrm{ml} /$ plate of the mix and were incubated, aerobically, at $37^{\circ} \mathrm{C}$. After 24 and 48 hours of incubation, each plate was examined and the colonies of B. anthracis and of contaminants were counted. B. anthracis colonies were identified by anthrax-specific PCRs [17].

\section{Statistical analysis}

The comparison between GABRI and standard methods, applied to the soil samples artificially and naturally contaminated, was carried out using the method of Bland and Altman [18].

\section{Results}

The results of GABRI and classic method applied on artificially and naturally anthrax-contaminated soil samples are shown in Tables 1 and 2, respectively. Table 2 reports the results of soil samples, purposefully contaminated with anthrax, evaluated by the classic method at three dilution levels and by the GABRI method. As shown, no anthrax spores were detected in these samples using the classic procedure, even when undiluted suspensions were examined; in contrast, all samples were positive to the GABRI method. With regard to contaminants, the GABRI method revealed a microbial contamination averaging nearly 1.1 colonies per plate, while by using the classic method, the microbial contamination averaged 59.7 colonies per plate in the suspension, 22.2 in the 1:10 dilution and 3.1 in the 1:100 dilution (Table 2).

Table 1 reports the results of naturally contaminated soil samples from Bangladesh, evaluated by both methods. As shown, when these samples were tested by the classic method, spores of $B$. anthracis were detected only in four undiluted samples, in three samples diluted 1:10 and in two samples diluted 1:100. In contrast, all samples resulted positive to GABRI method. This method revealed a microbial contamination averaging nearly 55 colonies per plate, while the classic method averaged 297 colonies per plate in the suspension, 56 in the 1:10 dilution and 7 in the 1:100 dilution (Table 1).

\section{Discussion}

The results confirmed that the GABRI method was more efficient than the classic method in detecting anthrax spores even in samples with low level of B. anthracis contamination.

Interesting is the result concerning the reduction of the microbial contaminants: in the anthrax spore contaminated soil samples, the presence of contaminants was significantly reduced when GABRI method was used respect to the classic method (Tables 1 and 2). This result is significant considering that in the GABRI a suspension volume of $1 \mathrm{ml}$ was tested while the classic method a volume of $0.1 \mathrm{ml}$ was examined. 
Table 2 Purposefully anthrax spore-contaminated soil samples examined by the classic method at three dilution levels and by the GABRI method

\begin{tabular}{|c|c|c|c|c|c|c|c|c|c|}
\hline \multirow{3}{*}{$\begin{array}{c}\text { Soil } \\
\text { sample }\end{array}$} & \multirow{3}{*}{$\begin{array}{l}\text { Anthrax } \\
\text { spores added } \\
\text { to sample }\end{array}$} & \multirow{2}{*}{\multicolumn{3}{|c|}{$\begin{array}{c}\text { CFU of } B \text {. anthracis } \\
\text { isolated by classic method } \\
\text { Total of } 10 \text { plates }\end{array}$}} & \multirow{2}{*}{\multicolumn{3}{|c|}{$\begin{array}{c}\begin{array}{c}\text { CFU of contaminants } \\
\text { isolated by classic method }\end{array} \\
\text { Total of } 10 \text { plates }\end{array}$}} & \multirow{2}{*}{\multicolumn{2}{|c|}{$\begin{array}{c}\text { CFU of } B \text {. anthracis and contaminants } \\
\text { isolated by GABRI method }\end{array}$}} \\
\hline & & & & & & & & & \\
\hline & & Undiluted & $1: 10$ & 1:100 & Undiluted & $1: 10$ & $1: 100$ & CFU of $B$. anthracis & CFU of contaminants \\
\hline N.1 & 520 & 0 & 0 & 0 & 725 & 341 & 124 & 2 & 8 \\
\hline N.2 & 480 & 0 & 0 & 0 & 714 & 337 & 8 & 2 & 9 \\
\hline N.3 & 500 & 0 & 0 & 0 & 1000 & 289 & 54 & 2 & 3 \\
\hline N.4 & 570 & 0 & 0 & 0 & 225 & 45 & 1 & 6 & 4 \\
\hline N.5 & 430 & 0 & 0 & 0 & 334 & 29 & 1 & 4 & 15 \\
\hline N.6 & 500 & 0 & 0 & 0 & 584 & 292 & 2 & 3 & 27 \\
\hline Average & 500 & 0 & 0 & 0 & 597 & 222.2 & 31.6 & 3.2 & 11.0 \\
\hline
\end{tabular}

The statistical comparison between the two methods was carried out using the method of Bland Altman, through which it was observed that the two methods are not statistically similar (Figure 1). The GABRI method produces a measure of the presence of contaminants significantly different from the classic method. In particular, the classic method is more sensitive to the presence of other Bacillus spp., while the GABRI method is more sensitive to the presence of Bacillus anthracis compared to the classic method.

To improve the efficiency of classical procedures for detection of anthrax spores in environmental samples, we evaluated a new microbiologic method which in

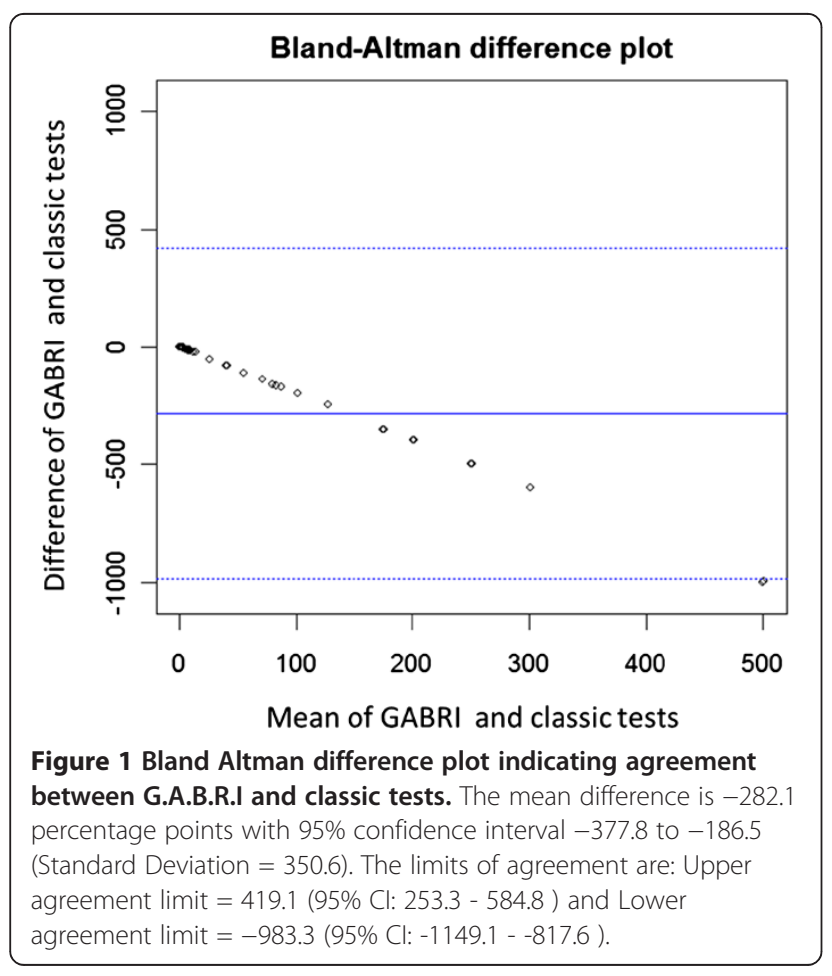

preliminary tests proved to be sensitive and able to distinguish B. anthracis from other ubiquitous species.

When environmental samples are tested for the presence of anthrax spores, the main problems are efficiently separating Bacillus spores from soil particles and strongly reducing the presence of contaminants which being much more numerous than $B$. anthracis, tend to either inhibit the development of anthrax organisms or just make it extremely difficult to accurately read the cultured result.

The contamination with vegetative cells is not a problem, since they can be easily eliminated by treating samples at temperatures that are lethal for vegetative microbes but not for spores. It has been demonstrated that Bacillus spores are hydrophobic and that they adhere to solid matrices especially by mean of hydrophobic interactions [19].

In the GABRI method soil samples were washed for a long time (30 min) with a wash buffer containing $0.5 \%$ of Tween 20. As previously demonstrated, in fact, the non-ionic detergents, such as Triton or Tween, allow the separation of spores from soil particles by disrupting hydrophobic interactions with solid matrices [9]. After washing with Tween 20, anthrax spores were recovered from supernatant by centrifugation.

To reduce the presence of contaminants, we treated soil samples with fosfomycin. To evaluate the environmental behavior of B. anthracis, Schuch and Fischetti investigated on the role of bacteriophages on bacterial adaptive behavior and niche expansion. In their study, the phage proteins encoding for fosfomycin resistance were specifically described in the spore surface structure of $B$. anthracis. Genes encoding surface proteins and antibiotic resistance may not be virulence factors in the classic sense but can help B. anthracis better survive within the highly competitive soil environment [20].

Based on these findings, in the GABRI method supernatants of soil samples, containing anthrax spores, were 
incubated with fosfomycin $(50 \mu \mathrm{g} / \mu \mathrm{l})$ prior to being plated onto selective medium. The results indicated that the treatment with fosfomycin strongly reduced microbial contaminants: the number of colonies of contaminants isolated in plates after GABRI method was significantly inferior to that obtained with classic method (Tables 1 and 2).

The reduced impact of the microbial environment allows the sowing of a larger quantity of a suspension and the isolation of anthrax organisms when they are present in very low concentrations in the soil. $B$. anthracis was isolated from $100 \%$ of artificially or naturally contaminated soil samples tested by the GABRI method; in contrast, $43 \%$ and $100 \%$ of naturally and artificiallycontaminated samples, respectively, gave negative results when evaluated by the classic method.

In the classic method usually some $100 \mu \mathrm{l}$ of the suspension is sown as is and reading these plates can be very difficult. In fact, in the absence of inhibiting actions, the microbial environment is essentially unchanged and the resulting thick carpet of bacteria makes the observation of any $B$. anthracis colonies very difficult, if not impossible. Previous experiments conducted in our laboratory on artificially contaminated soils have confirmed the reduction of the environmental contaminants up to $99 \%$ (unpublished data).

\section{Conclusions}

Our results indicate that, due to its ability to strongly reduce contaminants, the GABRI method may be especially suitable for environmental investigations.

Although the GABRI method makes it possible to isolate $B$. anthracis in environmental samples at very low levels of contamination, it should be overemphasized that the most important part of the entire process is the collecting phase. An essential aspect is the collaboration with the farmers because they can give useful, sometimes very accurate information on the actual places where the animals were slaughtered or buried. Moreover, for the pastures considered "infected", the period of the year when to optimally collect the samples is very important. In regard to historic retrospective investigations we generally recommend that the soil sampling is done in the fall or winter as the pasture grass is short and therefore one can make a better assessment of the orography of the investigated site. The weather conditions are important too. If the soil sampling is done immediately after rain, one has the possibility of taking samples of mud puddles that can appear on an otherwise anonymous slope; these "puddles" can mark the site(s) of cattle graves whose exact location is long forgotten. This system was adopted in Tuscany (Italy) on pastures where years before there had been outbreaks of anthrax in farm cattle. It is necessary to analyze the sample three or four times before declaring it negative.

\section{Authors' contributions}

AF: Designed, carried out and evaluated all the experimental studies conducted in ABL3 facilities. PDT: collaborated to the experimental studies conducted in ABL3 facilities; GG: carried out molecular assays to identify $B$. anthracis colonies; VC: carried out statistical analysis; LM: collaborated to the experimental studies conducted in ABL3 facilities; DB: collaborated to the experimental studies conducted in ABL3 facilities; CP: prepared all media for culturing and isolation of $B$. anthracis; RA: revised the experimental design and collaborated on the report of the manuscript; $\mathrm{MHJ}$ : revised the experimental design and collaborated on the report of the manuscript. All authors read and approved the final manuscript.

\section{Author details}

${ }^{1}$ Istituto Zooprofilattico Sperimentale della Puglia e della Basilicata, Anthrax Reference Institute of Italy, Foggia, Italy. ${ }^{2}$ Istituto Zooprofilattico Sperimentale dell'Abruzzo e del Molise "G. Caporale", Teramo, Italy. ${ }^{3}$ Departement of Veterinary Medicine, University of Bari, Bari, Italy. ${ }^{4}$ Istituto Superiore di Sanità, Roma, Italy. ${ }^{5}$ Department of Environmental Sciences School of the Coast \& Environment, Louisiana State University, Baton Rouge, LA 70803-5703, USA.

Received: 4 April 2013 Accepted: 3 July 2013

Published: 18 July 2013

\section{References}

1. WHO: Integrated control of neglected zoonotic diseases in Africa: applying the 'One healt Concept'. Geneva: WHO Document Production Services; 2009.

2. Smith $K L$, DeVos V, Bryden H, Price LB, Hugh-Jones ME, Keim P: Bacillus anthracis diversity in Kruger National Park. J Clin Microbiol 2000, 38(10):3780-3784.

3. Higgins $\mathrm{CH}$ : Anthrax. In Health of Animals Branch, Bulletin 23. Ottawa: Department of Agriculture; 1916:3-8.

4. Minett FC, Dhanda MR: Multiplication of Bacillus anthracis and Cl. Chauvoei in soil and water. Indian journal of veterinary science and animal husbandry 1941, 11:308-321.

5. Van Ness G, Stein CD: Soils of the United States favorable for anthrax. J Am Vet Med Assoc 1956, 128(1):7-12.

6. Van Ness GB: Ecology of anthrax. Science 1971, 172(3990):1303-1307.

7. Hugh-Jones M, Blackburn J: The ecology of Bacillus anthracis. Molecular aspect of medicine 2009, 30(6):356-367.

8. Turnbull PC: Definitive identification of Bacillus anthracis-a review. J App/ Microbiol 1999, 87(2):237-240

9. Dragon DC, Rennie RP: Evaluation of spore extraction and purification methods for selective recovery of viable Bacillus anthracis spores. Lett Appl Microbiol 2001, 33:100-105.

10. Marston CK, Beesley C, Helsel L, Hoffmaster AR: Evaluation of two selective media for the isolation of Bacillus anthracis. Lett Appl Microbiol 2008, 47(1):25-30.

11. Gulledge JS, Luna VA, Luna AJ, Zartman R, Cannons AC: Detection of low numbers of Bacillus anthracis spores in three soils using five commercial DNA extraction methods with and without an enrichment step. $J$ Appl Microbiol 2010, 109(5):1509-1520.

12. Ryu C, Lee $K$, Yoo C, Seong WK, Oh HB: Sensitive and rapid quantitative detection of anthrax spores isolated from soil samples by real-time PCR. Microbiol Immunol 2003, 47(10):693-699.

13. Fasanella A, Garofolo G, Hossain MJ, Shamsuddin M, Blackburn JK, HughJones M: Bangladesh anthrax outbreaks are probably caused by contaminated livestock feed. Epidemiol Infect 2012, 20:1-8.

14. Fasanella A, Scasciamacchia S, Garofolo G: The behaviour of virulent Bacillus anthracis strain A0843 in rabbits. Vet Microbiol 2009, 133:208-209.

15. Office International des Epizooties: Manual of Diagnostic Tests and Vaccines for Terrestrial Animals. 5th edition. Paris, France: OIE.

16. Turnbull PC, Frawley DA, Bull RL: Heat activation/shock temperatures for Bacillus anthracis spores and the issue of spore plate counts versus true numbers of spores. J Microbiol Methods 2007, 68(2):353-357.

17. Fasanella A, Losito S, Trotta T, Adone R, Massa S, Ciuchini F, Chiocco D: Detection of anthrax vaccine virulence factors by polymerase chain reaction. Vaccine 2001, 19(30):4214-4218. 
18. Bland JM, Altman DG: Statistical methods for assessing agreement between two methods of clinical measurement. Lancet 1986, 327(8476):307-310. doi:10.1016/50140-6736(86)90837-8.

19. Rönner U, Husmark U, Henriksson A: Adhesion of bacillus spores in relation to hydrophobicity. J Appl Bacteriol 1990, 69(4):550-556

20. Schuch R, Fischetti VA: The secret life of the anthrax agent bacillus anthracis: bacteriophage-mediated ecological adaptations. PLoS One 2009, 4(8):e6532.

doi:10.1186/1471-2180-13-167

Cite this article as: Fasanella et al:: Ground Anthrax Bacillus Refined

Isolation (GABRI) method for analyzing environmental samples with low

levels of Bacillus anthracis contamination. BMC Microbiology 2013 13:167.

\section{Submit your next manuscript to BioMed Central and take full advantage of:}

- Convenient online submission

- Thorough peer review

- No space constraints or color figure charges

- Immediate publication on acceptance

- Inclusion in PubMed, CAS, Scopus and Google Scholar

- Research which is freely available for redistribution 